\title{
A Retrospective Analysis of Toxicity and Efficacy for 2 Hypofractionated Irradiation Schedules Versus a Conventional One for Post-Mastectomy Adjuvant Radiotherapy in Breast Cancer
}

\author{
Vassilis Kouloulias $^{a, b} \quad E$ Efychia Mosa ${ }^{a}$ Anna Zygogianni ${ }^{b} \quad$ Efrosini Kypraiou $^{a}$ \\ John Georgakopoulos ${ }^{a}$ Kalliopi Platoni ${ }^{a}$ Christos Antypas $^{b}$ George Kyrgias ${ }^{c}$ \\ Maria Tolia ${ }^{b, c}$ Christos Papadimitriou $^{d}$ Amanda Psyrri $^{\mathrm{e}}$ George Patatoukas $^{a}$ \\ Maria Dilvoi $^{a}$ Christina Armpilia $^{\mathrm{b}}$ Kyriaki Theodorou $^{c} \quad$ Maria-Aggeliki Kalogeridic $^{\mathrm{c}}$ \\ Ivelina Beli ${ }^{\mathrm{a}}$ John Kouvaris ${ }^{\mathrm{b}}$ Nikolaos Kelekis $^{\mathrm{a}}$ \\ ${ }^{a}$ Radiotherapy Unit, 2nd Department of Radiology, Medical School, ATTIKON University Hospital, Athens, Greece; \\ ${ }^{b}$ Radiotherapy Unit, 1st Department of Radiology, Medical School, Aretaieion University Hospital, Athens, Greece; \\ c Radiotherapy Department, Medical School of Thessaly, University Hospital of Larisa, Larisa, Greece; \\ ${ }^{\mathrm{d}}$ Therapeutics Clinic, Alexandra Hospital, Medical School of Athens, Athens, Greece; \\ e Medical Oncology Unit, ATTIKON University Hospital, Athens, Greece
}

\section{Keywords}

Breast cancer - Postmastectomy irradiation .

Hypofractionation · Toxicity · Efficacy

\section{Summary}

Introduction: The aim of this analysis was a retrospective evaluation of the efficacy and toxicity of 2 hypofractionated irradiation schedules compared to conventional therapy in post-mastectomy patients. Methods: 3 irradiation schedules were analyzed: $48.30 \mathrm{~Gy}$ in 21 fractions (group $A$, $\mathrm{n}=60$ ), $42.56 \mathrm{~Gy}$ in 16 fractions (group $B, \mathrm{n}=27$ ) and $50 \mathrm{~Gy}$ in 25 fractions (group $C, n=30$ ) of the front chest wall. All groups were also treated with a supraclavicular field, with $39.10 \mathrm{~Gy}$ in 17 fractions (group A), 37.24 Gy in 14 fractions (group B) or $45 \mathrm{~Gy}$ in 25 fractions (group C). Results: No local recurrences were noted in any group during 36 months of follow-up. Acute skin toxicity presented in all groups, with $58.3 \%, 70.4 \%$ and $60 \%$ of grade I; $35 \%, 25.9 \%$ and $40 \%$ of grade II; $6.7 \%, 3.7 \%$ and $0 \%$ of grade III being seen in groups $A, B$ and $C$, respectively. Late skin toxicity was noted only as grade $I$ in $16.7 \%, 25.9 \%$ and $26.7 \%$ of groups A, B and C, respectively. No significant difference was noted among all groups for either acute or late skin toxicity, or for radio-pneumonitis (chi ${ }^{2}$ test, $p>0.05$ ). Conclusion: All schedules were equally effective with equivalent toxicity. A prospective randomized study is needed to confirm our results.

(๑) 2016 S. Karger GmbH, Freiburg

\section{Introduction}

For patients with operable breast cancer undergoing mastectomy, radiation therapy of the chest wall has been an important source for clinical radiobiological data. Literature supports the use of a variety of normal tissue endpoints in the radiobiological analysis [1]. The conventional dose of post-mastectomy radiotherapy is 5,000 cGy in 5 weeks, delivering 200 cGy daily, 5 days a week. However, due to huge work load, a hypofractionated protocol with the same radiobiological equivalent might be the solution for reduction of both the number of fractions and the total treatment duration. Our institution has already reported on hypofractionated radiotherapy in breast cancer in relation to irradiation after conservative surgery [2-5]. The aim of the present study was to investigate the potential efficacy, acute toxicity and long-term side effects of hypofractionated schedules.

\section{Material and Methods}

Here we report the results of a retrospective study of post-mastectomy radiation therapy in breast cancer patients performed at our institutions. Between 2008 and 2011, all patients $(\mathrm{n}=117)$ undergoing mastectomy at the ATTIKON, Aretaieion, or Larisa university hospitals received adjuvant radiotherapy, employing either hypofractionated schedules or the classic (200 cGy daily dose) radiotherapy scheme. Patients who were unable to undergo the conventional irradiation daily schedule of 5 weeks for various reasons (e.g. living in remote areas outside Athens) received the hypofractionated schedule; 60 were treated with $21 \times 230 \mathrm{cGy}$ (group A), 27 with $16 \times 266 \mathrm{cGy}$ (group B), and 30 with

\section{KARGER}

(C) 2016 S. Karger GmbH, Freiburg
Dr. Vassilios Kouloulias 
Table 1. Patients characteristics $(\mathrm{n}=117)$. All patients had invasive ductal carcinoma

\begin{tabular}{|c|c|c|c|c|c|}
\hline & \multicolumn{3}{|l|}{ Group } & \multirow[b]{2}{*}{ Total } & \multirow[b]{2}{*}{$\mathrm{p}$} \\
\hline & A & $\mathrm{B}$ & $\mathrm{C}$ & & \\
\hline $\mathrm{n}$ & 60 & 27 & 30 & 117 & \\
\hline Median age, years (range) & $57(33-73)$ & $60(34-76)$ & $54.5(37-74)$ & $57(33-78)$ & $0.72^{* *}$ \\
\hline \multicolumn{6}{|l|}{ T stage, $\mathrm{n}(\%)$} \\
\hline 2 & $11(18.3)$ & $4(14.8)$ & $7(23.3)$ & $22(18.8)$ & \multirow[t]{3}{*}{$0.78^{*}$} \\
\hline 3 & $44(73.3)$ & $20(74.1)$ & $22(73.3)$ & $86(73.5)$ & \\
\hline 4 & $5(8.3)$ & $3(11.1)$ & $1(3.3)$ & $9(7.7)$ & \\
\hline \multicolumn{6}{|l|}{$\mathrm{N}$ stage, n (\%) } \\
\hline 1 & $35(58.3)$ & $19(70.4)$ & $22(73.3)$ & & \multirow[t]{2}{*}{$0.29^{*}$} \\
\hline 2 & $25(41.7)$ & $8(29.6)$ & $8(26.7)$ & & \\
\hline Postmenopausal patients, n (\%) & $48(80.0)$ & $22(81.5)$ & $23(76.7)$ & $93(79.5)$ & $0.895^{\star}$ \\
\hline \multicolumn{6}{|l|}{ Hormone receptors status, $\mathrm{n}(\%)$} \\
\hline $\mathrm{ER}^{+}$and/or $\mathrm{PR}+$ & $55(76.7)$ & $25(92.6)$ & $29(96.7)$ & $109(93.2)$ & \multirow[t]{2}{*}{$0.429^{\star}$} \\
\hline $\mathrm{ER}-$ and $\mathrm{PR}^{-}$ & $5(8.3)$ & $2(7.4)$ & $1(3.3)$ & $8(6.8)$ & \\
\hline \multicolumn{6}{|l|}{ HER2neu status, n (\%) } \\
\hline+ & $25(41.7)$ & $10(37.1)$ & $12(40.0)$ & $47(40.2)$ & \multirow[t]{2}{*}{0.92} \\
\hline- & $35(58.3)$ & $17(62.9)$ & $18(60.0)$ & $70(59.8)$ & \\
\hline \multicolumn{6}{|l|}{ Ki67 status, n (\%) } \\
\hline$<12 \%$ & $42(70)$ & $15(55.6)$ & $22(73.3)$ & $79(67.5)$ & \multirow[t]{2}{*}{0.31} \\
\hline$>12 \%$ & $18(30.0)$ & $12(44.4)$ & $8(26.7)$ & $38(32.5)$ & \\
\hline
\end{tabular}

$\mathrm{ER}=$ Estrogen receptor, $\mathrm{PR}=$ progesterone receptor, HER2neu = human epidermal growth factor receptor.

${ }^{*}$ chi2 test; ${ }^{* *}$ Kruskall-Wallis test.

$25 \times 200 \mathrm{cGy}$ (group C) daily for 5 days a week. The selection of each scheme was related to the dose distributions and, when necessary (due to the maxima in front chest wall), the dose per fraction was decreased gradually according to treatment planning to either 2.3 Gy or 2 Gy from 2.66 Gy per fraction. Patients' characteristics are shown in table 1 .

The pretreatment evaluation included specific pathology review, and clinical examination. In addition, all patients underwent laboratory studies with complete blood count, chemistries and radiological staging with computed tomography (CT) of thorax and abdomen and bone scanning. Patients were staged using the TNM classification system [6].

Inclusion criteria were female patients of 33-78 years of age, T2-4 primary lesion and N1, N2, N3, or Nx nodal status. Post-mastectomy status evaluation, with axillary dissection was necessary. Exclusion criteria were breast conservation surgery, previous radiotherapy of the actual chest wall, and history of other malignant disease other than cervical cancer in situ or bilateral breast cancer. All patients had received the same chemotherapy: Adriamycin $60 \mathrm{mg} / \mathrm{m}^{2}$, cyclophosphamide $600 \mathrm{mg} / \mathrm{m}^{2}$ followed by weekly paclitaxel and started radiotherapy 20-40 days after the completion of chemotherapy. Informed consent was obtained from all patients prior to participation in this study. This retrospective study was approved by the local ethical committee.

\section{Radiotherapy}

The 3D treatment planning was based on CT images with 3-mm spacing from the apex of the lungs to the diaphragm including the whole lung and breast. The CT datasets were transferred either to the Prosoma ${ }^{\circledR}$ or ONCENTRA $^{\circledR}$ (ELECTA) virtual simulation and contouring system through the DICOM network. All contouring of target volumes and normal structures (organs at risk, OAR) were performed according to the ICRU criteria $[7,8]$.

The patients were irradiated according to 1 of 3 schedules: group A, $48.3 \mathrm{~Gy}$ (2.3 Gy in 21 fractions); group B, $42.56 \mathrm{~Gy}$ (2.66 Gy in 16 fractions); and group C, 50 Gy (2 Gy in 25 fractions; conventional schedule).

Concerning the irradiated volume for regional lymph nodes, all patients received radiation therapy from the middle side of level II of the axilla (where the surgeon stopped) up to the supraclavicular site (level IV) with the inclusion of level III of axilla. No radiotherapy was given to level I of axilla. All women also underwent radiotherapy with a supraclavicular beam and with a dose of 3,910 cGy in 17 fractions (group A), a dose of 3,724 Gy in 14 fractions (group B) or a dose 4,500 cGy in 25 fractions (group C).

We used the QUANTEC trial to determine the dose constraints for choosing normalized total dose (NTD) values (related to the hypofractionated schedules) for an $\alpha / \beta=3$ (late reacting tissues) [9]. Dose calculations were performed using either the treatment planning system Eclipse (Varian, Palo Alto, CA) or the ONCENTRA (ELECTA), to deliver the prescribed dose according to the International Commission on Radiation Units and Measurements (ICRU) reference point, in terms of $95-107 \%$ as a dose range $[10,11]$. An upper limit of $110 \%$ was set for avoiding any adverse events regarding radiation-induced toxicity. Histograms were generated for the treatment technique, with a number of parameters, including mean, median and maximum dose being evaluated. Patient setup was monitored weekly using portal films. The transformation of physical to radiobiological dose volume histograms for normal tissues was performed with the Niemierko model according to Thrapsanioti et al. [12]. All patients were treated on a VARIAN CLINAC 600C or SIEMENS Oncore or ELECTA SL-75 linear accelerator, with $6 \mathrm{MV}$ photon beams.

\section{Radiobiological Calculations}

We used linear-quadratic modeling to equate the hypofractionation schedules to the NTD when delivered in 2-Gy fractions [13-19]. Thus, NTD represents the dose given in 2-Gy fractions that would give the equivalent biological effect to the new hypofractionated dose: NTD $=D_{\text {new }}\left[\left(d_{\text {new }}+\alpha / \beta\right) /(2+\alpha / \beta)\right]$, where Dnew and dnew are the total dose and dose per fraction, respectively, for a suggested hypofractionation scheme. NTD has been calculated and tabulated for both breast $(\alpha / \beta=4 \mathrm{~Gy})$ and acute reacting tissues $(\alpha / \beta=10 \mathrm{~Gy})$ [13-19].

\section{Front Chest Wall Calculation}

For group $A$, for $\alpha / \beta=4$, the NTD was 50.72 Gy for 21 fractions; for $\alpha / \beta=10$, the NTD was 49.51 Gy for 21 fractions. For group $B$, for $\alpha / \beta=4$, NTD was 47.24 Gy for 16 fractions; for $\alpha / \beta=10$, the NTD was $44.9 \mathrm{~Gy}$. 
Table 2. Radiation-induced skin toxicity according to EORTC/RTOG criteria. No significant difference was noted for either acute $(\mathrm{p}=0.492$, chi $^{2}$ test $)$ or late $\left(\mathrm{p}=0.444\right.$, chi $^{2}$ test $)$ toxicity

\begin{tabular}{clll}
\hline & Group A & Group B & Group C \\
& 2.30 Gy/fraction & 2.66 Gy/fraction & 2 Gy/fraction \\
\hline n & 60 & 27 & 30 \\
$\begin{array}{l}\text { EORTC/RTOG skin toxicity } \\
\text { Acute, n (\%) } \\
\text { Grade 1 }\end{array}$ & & & \\
Grade 2 & $35(58.3)$ & $19(70.4)$ & $18(60)$ \\
Grade 3 & $21(35)$ & $7(25.9)$ & $12(40)$ \\
Late, n (\%) & $4(6.7)$ & $1(3.7)$ & - \\
Grade 0 & & & $22(73.3)$ \\
Grade 1 & $50(83.3)$ & $20(74.1)$ & $8(26.7)$ \\
\hline
\end{tabular}

\section{Supraclavicular Region}

For group A, for $\alpha / \beta=4$, the NTD was 50.72 Gy for 21 fractions; for $\alpha / \beta=10$, the NTD was $49.51 \mathrm{~Gy}$ for 21 fractions. For group B, for $\alpha / \beta=4$, NTD was $47.24 \mathrm{~Gy}$ for 16 fractions; for $\alpha / \beta=10$, the NTD was $44.9 \mathrm{~Gy}$. For Group $C$, the schedule was 23 fractions with 2 Gy per fraction (conventional scheme).

\section{Endpoints}

Endpoints included treatment feasibility, acute and late safety outcomes, cosmetic results and short-term efficacy. Treatment feasibility was defined as the successful delivery of the prescribed dose following the intended treatment schedule. Efficacy was based on the rate of local recurrence. Acute and late radiation-induced skin toxicity grading was performed according to EORTC/ RTOG toxicity criteria [18]. According to the procedure followed, all patients underwent echocardiography before and after radiotherapy. A fall of more than $10 \%$ in ejection fraction (EF) was taken as a significant reduction in the left ventricular EF whether symptomatic or not. Patients with a baseline EF of $<55 \%$ were excluded from the study. Lung CT scans for assessing any pulmonary toxicity were performed before treatment and 4 weeks and 6 months after radiotherapy. Pulmonary toxicity was evaluated according to a scale developed at our Institution [19]. Any injury of the brachial plexus causing arm damage and weakness was also documented with neurological examination. Lymphedema was measured as the circumference at the middle of the forearm. The highest difference during follow-up was taking as the final measurement. The grading scale used for scoring lymphedema was: grade 0 (no difference), grade I (increase of $0-1 \mathrm{~cm}$ ), grade II (increase of 1-2 cm) and grade III (increase $>2 \mathrm{~cm}$ ). All patients were evaluated weekly during treatment and reviewed every month later on, after the completion of the radiotherapy, to assess acute/late toxicities.

Data at diagnosis (baseline), at the end of radiation treatment and from all monthly follow-up visits, up to 6 months after treatment were analyzed in this study. Symptoms occurring between the start of radiotherapy and 90 days after this time point were classified as 'acute'. Symptoms occurring 6 months after the end of treatment were defined as 'late'. Toxicity grading was performed according to EORTC/RTOG criteria [18].

\section{Statistical Methods}

Local recurrence-free rates were calculated from the onset of the radiotherapy. Relapse-free survival was calculated using Kaplan-Meier method. Comparison of mean values among the different groups was performed with the Kruskall-Wallis non-parametric test. The evaluation of the differences in the incidence of acute or late radiation-induced toxicity, radio-pneumonitis, estrogen/progesterone receptor status and menopausal status among the irradiated groups was performed with the Pearson chi $^{2}$ test. The significance level was set at 0.05 . The analysis was performed with the SPSS ver. 10 software (IL, USA).

\section{Results}

All patients had good performance status according to Eastern Cooperative Oncology Group performance score of $0-1$. As shown in table 1, no significant differences in terms of patients' characteristics were noted among the 3 groups, confirming the homogeneity of data in the groups of patients. Median follow-up duration was 36 months. The intention was to keep the dose range between $95 \%$ and $107 \%$. Patients were initially evaluated for the 2.66 Gy per fraction; for dose distributions over $107 \%$, the final decision was either 2.3 Gy or 2 Gy per fraction.

The mean volumes of regional lymph nodes irradiated for groups A, B and C was $86.5 \pm 14.9,84.4 \pm 13.6$ and $83.1 \pm 14.7 \mathrm{cc}$, respectively. No significant difference was noted $(\mathrm{p}=0.23$, Kruskall-Wallis test).

There was no documentation of severe (grade 4) toxicities in any of the patients. After 3 years of follow-up, no local recurrences were observed. The incidence of acute and late complications among the studied groups is shown in table 2. In general, EORTC/ RTOG acute and late skin toxicity for the hypofractionated irradiation schedules showed no statistical difference between the 2 schedules ( $\mathrm{Chi}^{2}$ test). No hematological toxicity or rib fracture was observed. The mean values for acute EORTC/RTOG toxicity scores in groups A, B and C were: $1.48 \pm 0.62,1.33 \pm 0.55$ and $1.40 \pm 0.5$, respectively. The mean values for late EORTC/RTOG toxicity scores in groups A, B and C were: $0.17 \pm 0.38,0.26 \pm 0.45$ and $0.27 \pm 0.45$, respectively. In total, no statistical significance was noted between the 3 radiotherapy schedules in terms of either acute ( $\mathrm{p}=0.543$, Kruskall-Wallis) or late ( $\mathrm{p}=0.447$, KruskallWallis test) mean value of reported scores for radiation-induced skin toxicity .

Concerning lymphedema, only grade I lymphedema (but without significant difference, $\mathrm{p}=0.584, \mathrm{chi}^{2}$ test) was noted for groups $\mathrm{A}, \mathrm{B}$ and $\mathrm{C}$ as $81.6 \%, 85.2 \%$ and $90 \%$, respectively. No injury to the brachial plexus or weakness of the ipsilateral arm was noted during the follow-up. The incidence of radiation-induced pulmonary toxicity is shown in details in table 3 . No grade 3 or 4 lung toxicity was noted. No significant difference was assessed among the groups concerning the incidence of radiation pneumonitis $\left(\mathrm{p}=0.952\right.$, chi $^{2}$ test $)$. 
Table 3. Radiation-induced toxicity according to grading scale based on CT images [16]. No significant differences were noted ( $\mathrm{p}=0.952$, chi ${ }^{2}$ test). Grade 3 or 4 toxicity was not noted

\begin{tabular}{llll}
\hline & Group A & Group B & Group C \\
\hline $\mathrm{n}$ & 60 & 27 & 30 \\
Grade $0^{\mathrm{a}}, \mathrm{n}(\%)$ & $47(78.3)$ & $20(74.1)$ & $24(80)$ \\
Grade $1^{\mathrm{b}}, \mathrm{n}(\%)$ & $12(20.0)$ & $6(22.2)$ & $5(16.7)$ \\
Grade $2^{\mathrm{c}}, \mathrm{n}(\%)$ & $1(1.7)$ & $1(3.7)$ & $1(3.3)$ \\
\hline
\end{tabular}

${ }^{\mathrm{a}}$ No findings.

${ }^{\mathrm{b}}$ Ground glass opacities without fuzziness of the subjacent pulmonary vessels. ${ }^{c}$ Findings may vary from ground glass opacities, extending beyond the radiation field, to consolidations.

\section{Discussion}

Post-mastectomy radiotherapy has improved local regional control and overall survival [20-22]. In our trial, data analysis of all 3 groups showed similar results in all arms and enhances the hypothesis that larger fractions are equally effective in controlling the locoregional disease $[23,24]$. Regarding early toxicity, the appearance of erythema is a complex reaction, with various groups of target cells involved [25]. In the study of Eldeed et al. [26], 107 patients underwent different fractionation regimens. Group A (41 patients) received 50 Gy over 25 fractions, group B (36 patients) received $45 \mathrm{~Gy}$ in 17 fractions and group C (30 patients) received 40 Gy in 15 fractions. After a 7-year follow-up, the Egyptian trial demonstrated no significant difference in disease-free survival or overall survival between the groups. Similarly, we did not noticed any differences in our study and no recurrences have been noted after 3 years of follow-up.

In the study of Shuhid et al. [27] of 300 post-mastectomy patients with breast cancer stage T2-4, Nx were randomized to be irradiated with Co60 unit to either 2,700 cGy in 5 fractions (1 week) arm A, or 3,500 cGy in 10 fractions (2 weeks) arm B or 4,000 cGy in 15 fractions (3 weeks) arm C. The locoregional relapses were $11 \%$, $12 \%$ and $10 \%$ in arms A, B and C, respectively. $26 \%, 24 \%$ and $28 \%$ patients developed metastatic disease and $17 \%, 18 \%$ and $20 \%$ died in the 3 arms. G3 and G4 skin toxicities were 37\%, 28\% and $14 \%$. G2 and G3 lymphedoema was $21 \%, 22 \%$ and $27 \%$. Cardiac toxicity was $5 \%, 6 \%$ and $5 \%$, while pulmonary toxicity was $4 \%, 5 \%$ and $5 \%$, respectively. All the differences except skin toxicity were statistically insignificant. These results showed higher toxicity and relapses rate than in our study. The main reason for that might be the fact that Shuhid et al. used a Co6 unit for irradiation instead of LINAC, and a $2 \mathrm{D}$ technique instead of 3D conformal. Dose distributions with Co60 appear to be definitely inferior and inhomogeneous compared with that of the LINAC and the 3D conformal technique.

Regarding late reactions, telangiectasia is related to compensatory permanent dilation of the remaining capillaries, arterioles or venules [28]. An analysis of the Gothenburg teleangiectasia data showed that the incidence of severe teleangiectasia depended on overall treatment time [29]. Latency of teleangiectasia depends on the amount of tissue injury inflicted by the irradiation [30]. Previous studies have shown that the degeneration rate is determined during the first 3 weeks, when proliferation is assumed to be negligible, and has been found to be independent of fractionation and dose intensity [25]. Recovery, undisturbed by radiation, is very rapid. This means that even fairly short rest periods during the treatment effectively reduce the acute reactions. Thus, even with an accelerated hypofractionated scheme, the rate of teleangiectasia does not change dramatically.

In general, various clinical studies have shown that late radiation reactions are irreversibly progressing with time [31]. Regarding late reactions, there was no statistically significant difference between the three groups, while only grade I was demonstrated in our study. According to M.D. Anderson Cancer Center, very severe subcutaneous fibrosis and skin necrosis continue to occur even at 10 years of follow-up [32]. Thus, in our study, the 3 -years of follow-up might be short for any late skin toxicity to be noted. This is really the weakness of our study; however, follow-ups are ongoing and further results will be reported when follow-up reaches 5-7 years. As far as supraclavicular radiation fields are concerned, there are limited data available for the toxicity caused by hypofractionated radiation schedules. In our study, there was no significant difference in toxicity due to the hypofractionated radiotherapy compared with conventional fractionation of the supraclavicular area. This is in accordance with the results of a recent review published by Badiyan et al. [33]. More specifically, after the radiobiological transformation and examination of the OAR dose volume histograms, it was found that breast, skin and heart had no greater toxicity due to the shorter schedule. According to the scale of toxicity for radio-pneumonitis used in the present study, no significant difference was noted among the 3 groups.

Overall, the additional radiotherapy of the supraclavicular area may increase the rate of pneumonitis because of the larger volume of the lung that is exposed to therapy. However, when strict pulmonary constraints are used, the rates of pneumonitis are kept low [34]. Apart from this, supraclavicular irradiation may potentially cause brachial plexopathy, which is connected to dose per fraction, total dose and volume irradiation. However, several studies that delivered daily doses lower than 3 Gy and total doses of less than 45 Gy found that brachial plexopathy occurred at similar rates to those seen with conventional fractionation $[35,36]$. In our study, no plexopathy was noted in any of the groups, and no significant difference was assessed among the 3 groups with respect to the irradiated volume of regional lymph nodes. Moreover, after evaluating the irradiated volumes of regional lymph nodes, we found that these conformed to the relevant ESTRO guidelines, helping to reduce the potentially related radiation-induced toxicity [37].

In conclusion, our study has shown that $3 \mathrm{D}$ conformal radiation therapy is a feasible and safe modality, allowing for hypofractionation with either $2.30 \mathrm{~Gy}$ in 21 fractions or $2.66 \mathrm{~Gy}$ in 16 fractions in women after mastectomy. The 2 hypofractionated schedules were found to be as effective as the conventional schedule, achieving a high locoregional control rate, while the toxicity was mild and equivalent to that seen with the conventional scheme. It 
should be mentioned that this was a retrospective study with the danger of bias. Although the 2 proposed hypofractionated schedules can be recommended for adjuvant irradiation post mastectomy, a prospective randomized study needs to be performed to confirm our results. A follow-up of at least 5 years post irradiation is also mandatory to document any late reactions or potential relapses. However, due to the lack of powerful evidence, and mainly due to the need for further trials, we are sharing our experience to contribute to a brainstorming concerning the growing interest for hypofractionation in women after mastectomy.

\section{Acknowledgments}

We do wish to thank Dr. Alfred Barich for English language editing.

\section{Disclosure Statement}

The authors indicated no potential conflict of interest.

\section{References}

1 Zygogianni AG, Kouloulias V, Armpilia C, et al.: The potential role of hypofractionated accelerated radiotherapy to cosmesis for stage I-II breast carcinoma: a prospective study. J BUON 2011;16:58-63.

2 Zygogianni A, Kouloulias V, Kyrgias G, et al.: Comparison of two radiotherapeutic hypofractionated schedules in the application of tumor bed boost. Clin Breast Cancer 2013;13:292-298.

3 Zygogianni AG, Kouvaris JR, Kouloulias V, et al.: Hypofractionated accelerated irradiation for stage I-II breast carcinoma: A phase II study. Breast J 2010;16: 337-338.

4 Kalogeridi MA, Kelekis N, Kouvaris J, et al.: Accelerated hypofractionated radiotherapy schedules in breast cancer: A review of the current literature. Rev Recent Clin Trials 2009;4:147-151.

5 Tolia M, Platoni K, Foteineas A, et al.: Assessment of contralateral mammary gland dose in the treatment of breast cancer using accelerated hypofractionated radiotherapy. World J Radiol 2011;3:233-240.

6 American Joint Committee on Cancer (AJCC), Chicago, II. Cancer Staging Manual 7th ed. Springer, 2010

7 ICRU 50. Prescribing, recording and reporting photon beam therapy. Bethesda, MD, ICRU, 1993.

8 International Commission on Radiation Units and Measurements (ICRU).Report 62. Prescribing, recording, and reporting photon beam therapy (Supplement to ICRU Report 50). Bethesda, MD, ICRU, 1999.

9 Bentzen S, Constine L, Deasy J, et al.: Quantitative analyses of normal tissue effects in the clinic (QUANTEC): An introduction to the scientific issues. Int J Radiat Oncol Biol Phys 2010;76:3-9.

10 Thames HD, Bentzen SM, Turesson I, et al.: Time-dose factors in radiotherapy: A review of human data. Radiother Oncol 1990;19:219-235.

11 Fowler JF, Tome WA, Fenwick JD, Mehta MP: A challenge to traditional radiation oncology. Int J Radiat Oncol Biol Phys 2004;60:1241-1256.

12 Thrapsanioti Z, Karanasiou I, Platoni K, et al.: Transformation of physical DVHs to radiobiologically equivalent ones in hypofractionated radiotherapy analyzing dosimetric and clinical parameters: A practical approach for routine clinical practice in radiation oncology. Comput Math Methods Med 2013;2013:713420.

13 Fowler JF: The linear-quadratic formula and progress in fractionated radiotherapy. Br J Radiol 1989;62:679-694.
14 Emami B, Lyman J, Brown A, et al.: Tolerance of normal tissue to therapeutic irradiation. Int Radiat Oncol Biol Phys 1991;21:109-122.

15 Barendsen GW: Dose fractionation, dose rate, and isoeffect relationships for normal tissue responses. Int J Radiat Oncol Biol Phys 1982;8:1981-1997.

16 Dale RG: The application of the linear-quadratic doseeffect equation to fractionated and protracted radiotherapy. Br J Radiol 1985;58:515-528.

17 Thames HD: An 'incomplete-repair' model for survival after fractionated and continuous irradiations. Int J Radiat Biol 1985;47:319-339.

18 Cox JD, Stenz J, Pajak TF: Toxicity criteria of the radiation therapy oncology group (RTOG) and the European organization for research and treatment of cancer. Int J Radiat Oncol Biol Phys 1995;5:1341-1346.

19 Kouloulias V, Zygogianni A, Efstathopoulos E, et al.: Suggestion for a new grading scale for radiation induced pneumonitis based on radiological findings of computerized tomography: Correlation with clinical and radiotherapeutic parameters in lung cancer patients. Asian Pac J Cancer Prev 2013;14:2717-2722.

20 Killander F, Anderson H., Ryden S, et al.: Efficient reduction of loco-regional recurrences but no effect on mortality twenty years after mastectomy radiation in premenopausal women with stage II breast cancer-A randomized trial from the South Sweden Breast Cancer Group. Breast 2009;18:309-315.

21 Clarke M, Collins R, Dardy S, et al.: Effects of radiotherapy and of differences in the extent of surgery for early breast cancer on local recurrence and 15-year survival: an overview of the randomized trials. Lancet 2005;366:2087-2106.

22 Whealan T, Julian J, Wright J, et al.: Does locoregional radiation therapy improve survival in breast cancer? A meta-analysis. J Clin Oncol 2000;18:1220-1229.

23 Yarnold J, Ashton A, Bliss J, et al.: Fractionation sensitivity and dose response of late adverse effects in the breast after radiotherapy for early breast cancer, long term results of a randomized trial. Radiother Oncol 2005;75:9-17.

24 Yarnold J: Fractionation in radiotherapy: Results of a Canadian randomized clinical trial. Breast Cancer Online (www.bco.org) 2005;8(6).
25 Nyman J, Turesson I: Basal cell density in human skin for various fractionation schedules in radiotherapy. Radiother Oncol 1994;33:117-124.

26 Eldeed H, Awad I, Elhanafy O: Hypofractionation in post-mastectomy breast cancer patients: Seven-year follow-up. Med Oncol 2012;29:2570-2576.

27 Shahid A, Athar MA, Asghar S, et al.: Post mastectomy adjuvant radiotherapy in breast cancer: A comparision of three hypofractionated protocols. J Pak Med Assoc 2009;59:282-287.

28 Potten CS: Radiation and skin. Taylor and Francis, London, 1985, pp. 155-158.

29 Turesson I, Thames HD: Repair capacity and kinetics of human skin during fractionated radiotherapy: Erythema, desquamation and telangiectasia after 3 and 5 years follow-up. Radiother Oncol 1989;15:169-188.

30 Bentzen S, Turreson I, Thames HD: Fractionation sensitivity and latency of telangiectasia after postmastectomy radiotherapy: A graded-response analysis. Radiother Oncol 1990;18:95-106.

31 Thames HD, Bentzen SM, Turreson I, et al.: Fractionation parameters for human tissues and tumors. Int J Radiat Biol 1989;56:701-710.

32 Spanos WJ, Montague ED, Fletcher GH: Late complication of radiation only for the advanced breast cancer. Int J Radiat Oncol Biol Phys1980;6:1473-1476.

33 Badiyan SN, Shah C, Arthur D, et al.: Hypofractionated regional nodal irradiation for breast cancer: $\mathrm{Ex}$ aminating the data and potential for future studies. Radiother Oncol 2014;110:39-44.

34 Senthi S, Haasbeek CJA, Slotman BJ, Senan S: Outcomes of stereotactic ablative radiotherapy for central lung tumours: A systematic review. Radiother Oncol 2013;106:276-282.

35 Powell S, Cooke J, Parsons C: Radiation-induced brachial plexus injury: Follow up of two different fractionation schedules. Radiother Oncol 1990;18:213-220.

36 Soto O: Radiation-induced conduction block: Resolution following anticoagulant therapy. Muscle Nerve 2005;31:642-645.

37 Offersen BV, Boersma LJ, Kirkove C, et al.: ESTRO consensus guideline on target volume delineation for elective radiation therapy of early stage breast cancer. Radiother Oncol 2015;114:3-10. 